\title{
Building a cellular switch: more lessons from a good egg
}

\author{
James E. Ferrell, Jr.
}

\begin{abstract}
Summary
Xenopus oocytes mature in response to the steroid hormone progesterone. At the level of a population of oocytes, the response is graded - the higher the concentration of progesterone, the larger the fraction of oocytes that will mature-but at the level of the individual oocyte, the response is all-or-none. The all-or-none character of this cell fate switch is hypothesized to arise out of two properties of the signal transduction machinery that mediates maturation, positive feedback, and ultrasensitivity. This combination of positive feedback plus ultrasensitivity crops up again and again in cellular switches, from the lysis-lysogeny switch in phage-infected bacteria to the action potential in neurons. BioEssays 21:866-870, 1999.
\end{abstract}

(1) 1999 John Wiley \& Sons, Inc.

Earlier in this issue, I presented an overview the signal transduction system that triggers the maturation of Xenopus oocytes, the process through which a G2-arrested oocyte reenters meiotic $M$ phase and becomes a ripe egg, ready for fertilization. (1) Maturation is initiated by the binding of progesterone to an as yet poorly characterized receptor, which results in a decrease in cAMP levels and protein kinase $A$ activity. Eventually, the signal reaches the Mos/MEK1/p42 MAP kinase (MAPK) cascade. Through one or more mechanisms, activation of this cascade brings about activation of Cdc2. Cdc2 in turn seems to act positively on the MAPK cascade. These events are discussed in detail in the preceding review ${ }^{(1)}$ and several other recent reviews ${ }^{(2,3)}$ and are summarized schematically in Figure 1.

At the level of a population of oocytes, the maturation response is graded-as the concentration of progesterone increases, the proportion of oocytes that mature increases. However, even at low concentrations of progesterone, those oocytes that do mature, mature completely, and even at high concentrations of progesterone, those that do not mature, do not mature at all. Thus, at the level of the individual oocyte, the maturation response is all-or-none. This makes biological sense; under different environmental conditions, it may be

Department of Molecular Pharmacology, Stanford University School of Medicine, Stanford, California 94305-5332. E-mail: ferrell@cmgm. stanford.edu

Funding agency: National Institutes of Health: Grant numbers: GM46383; GM51233. appropriate for a frog to lay different numbers of eggs, but laying half mature eggs would be wasteful.

Thus, somehow the signal transducers that trigger maturation convert a graded stimulus, the concentration of progesterone, into an all-or-none cell fate decision. The "off state" of this cell fate switch must be stable-stage VI oocytes can remain arrested in their G2-like immature state essentially indefinitely-but once the oocyte commits to undergoing maturation, it must mature completely. Maturation must also be irreversible, so that after a maturation-inducing pulse of progesterone dissipates, the oocyte will not fall back from M-phase to interphase.

Even though at the level of the individual protein molecules, signal transducers like Cdc2 and p42 MAPK act as molecular switches, at the level of the whole oocyte, a Cdc2 response or MAPK response could be almost infinitely graded. The oocyte could have none of its p42 MAPK molecules active, or all $100 \times 10^{9}$ of its p42 MAPK molecules active, or any amount in between.

Here, I shall describe why the functional organization of these signal transducers into cascades and feedback loops is likely to generate the all-or-none, irreversible nature of oocyte maturation. Our interest in this question began with a separate issue, namely, why there are three kinases in the MAPK cascade when it seems like a single kinase might be able to accomplish the same thing.

\section{Magnitude amplification in the MAPK cascade?}

In principle, a kinase cascade like the MAPK cascade could achieve a tremendous degree of signal amplification; that is, a small concentration of active Mos could bring about the 


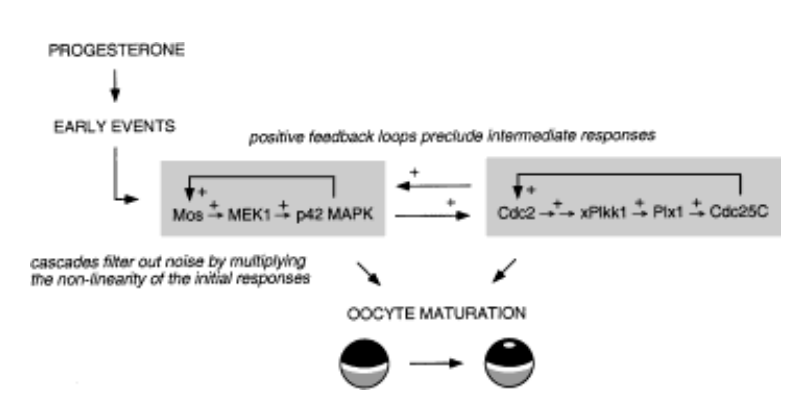

Figure 1. Schematic view of the signal transduction pathways that trigger Xenopus oocyte maturation.

activation of a larger concentration of MEK1, which could bring about the activation of a still larger concentration of $\mathrm{p} 42$ MAPK. However, in oocytes, the degree of amplification is modest, and not all of the steps in the cascade contribute. The synthesis of $\sim 10 \mathrm{nM}$ Mos causes the activation of much or all of the $1300 \mathrm{nM}$ MEK1 and $330 \mathrm{nM} \mathrm{p42} \mathrm{MAPK.(4)} \mathrm{Thus,}$ the overall amplification of the cascade is only $\sim 30$-fold, raising the possibility that there are important reasons other than magnitude amplification for triggering oocyte maturation by means of a cascade rather than a single protein kinase.

\section{Ultrasensitivity accumulates multiplicatively}

One possible reason for having a cascade (rather than a single signal transducer) trigger maturation-or more precisely, one potentially important consequence of a cascade arrangement-can be seen by considering how the stimulus/ response curves for the cascade kinases would be expected to change as the cascade is descended. If each individual level of a cascade exhibited a normal hyperbolic ("Michaelian") response to the kinase above it (Fig. 2A), the overall response of the cascade would be Michaelian as well. $(5,6)$ However, the situation would be different if individual levels of the cascade exhibited sigmoidal or "ultrasensitive" responses, responses resembling those of a cooperative enzyme. Because the responses of individual levels combine multiplicatively (see Appendix), the overall response of a cascade built out of ultrasensitive phosphorylation/dephosphorylation reactions could be very steeply sigmoidal and, in the extreme, approach a step-function (Fig. 2B). ${ }^{(5-7)}$

This in fact seems to be the case for the MAPK cascade in oocyte extracts. The steady state level of active MEK1 as a function of Mos concentration is well approximated by a Hill curve with a Hill coefficient of about 1.7 , and the steady state level of active p42 MAPK as a function of Mos exhibits a Hill coefficient of about $5^{(8)}$ (Fig. 3A,B). This is a very large Hill coefficient. It means that MAPK turns on the way a highly cooperative enzyme would; the first increments of stimulus produce very little response, and then once a threshold is
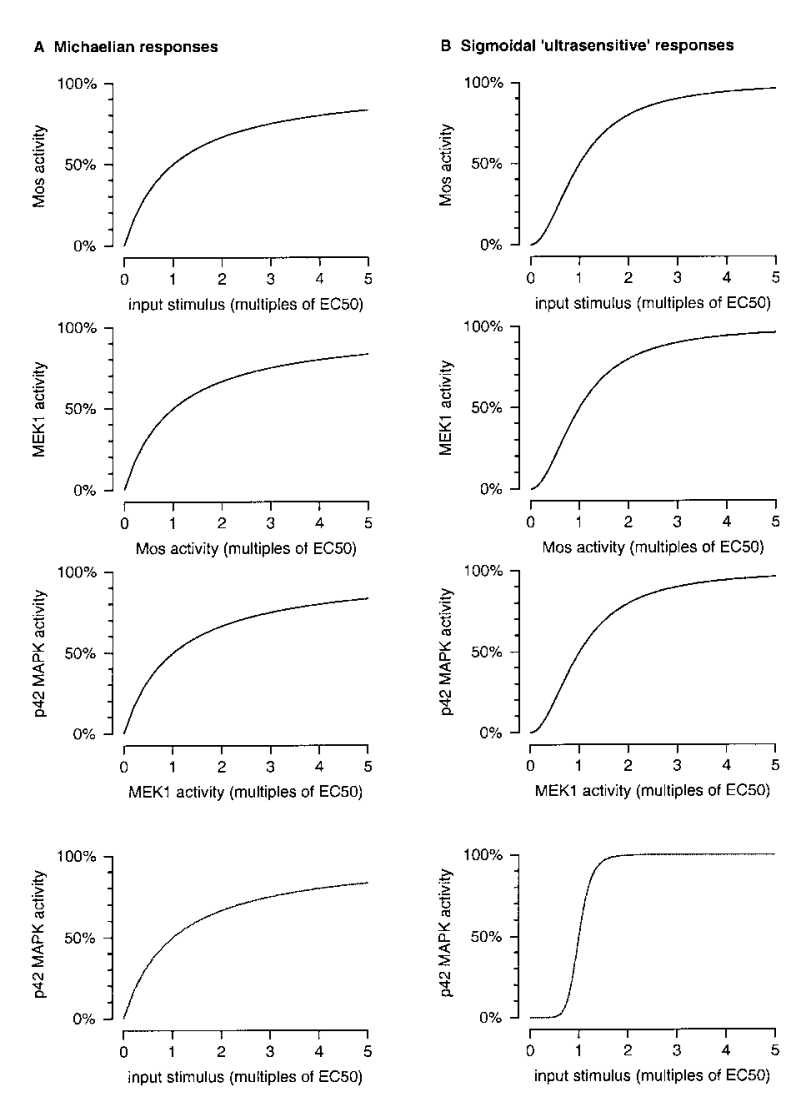

Figure 2. Steady-state responses combine multiplicatively in a signaling cascade. A: If the responses of the individual levels of a cascade are all Michaelian, the overall response of the cascade will be Michaelian as well. B: If the responses of the individual levels are sigmoidal or ultrasensitive, the overall response can be steeply sigmoidal.

reached, the MAPK begins to respond. Ultrasensitivity goes part way, but not all the way, toward explaining how the response of an oocyte to progesterone comes to be all-ornone in character.

\section{Sources of ultrasensitivity in the MAPK cascade}

The ultrasensitive response of MEK1 to Mos can be explained by the observation that the regulatory $\beta$-subunit of casein kinase II can physically interact with and inhibit Mos. ${ }^{(9,10)}$ Thus, the first Mos to accumulate will be relatively ineffective in activating MEK1, and only when Mos begins to exceed the capacity of this stoichiometric inhibitor will it become effective; the response of MEK1 to Mos should be sigmoidal, as is observed. The ultrasensitive response of p42 MAPK to MEK1 is thought to occur because it takes two phosphorylations to activate p42 MAPK and because the phosphorylations occur by a nonprocessive mecha- 


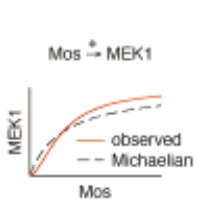
B
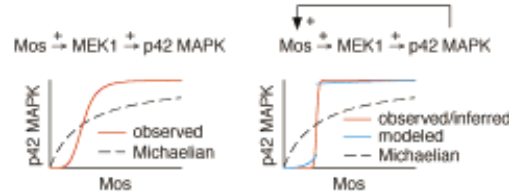

Figure 3. Building a switch. A, B: In the absence of feedback, the responses of MEK1 and p42 MAPK to Mos are sigmoidal or "ultrasensitive," with the p42 MAPK response (B) being more highly ultrasensitive than the MEK1 response (A). ${ }^{(8)}$ When positive feedback is permitted, the MAPK response becomes essentially all-or-none. C: The red curve represents a lower bound for how steep the responses of individual oocytes are, as inferred from experimental data. ${ }^{(18)}$ The blue curve is the modeled response for p42 MAPK activation in the presence of feedback. ${ }^{(18)}$

nism. ${ }^{4,8,11,12)}$ Other factors, in particular a phenomenon termed zero-order ultrasensitivity, probably contribute as well.(4)

Modeling studies have shown that, for a wide range of assumed concentrations and kinetic parameters, the MAPK cascade is predicted to exhibit ultrasensitivity; ${ }^{(8)}$ the prediction is robust. Thus, it seems likely that the MAPK cascade exhibits ultrasensitivity in other cell types and biological contexts. This hypothesis awaits experimental testing.

\section{Positive feedback in the MAPK cascade}

So far we have treated the MAPK cascade as a linear sequence of enzymes with Mos at the top and p42 MAPK at the bottom. But, as discussed in detail in the preceding review, ${ }^{(1)}$ in intact Xenopus oocytes, the cascade is embedded in a positive feedback loop. Not only can Mos bring about activation of p42 MAPK, but p42 MAPK can bring about accumulation of Mos, by stimulating the polyadenylation of the mos mRNA(13) and by increasing the stability of the Mos protein. ${ }^{(14-16)}$ This positive feedback loop does not operate in oocyte extracts-the Mos protein is apparently not translated at an appreciable rate(17)_but in the intact cell it does operate, and modeling studies show that a feedback loop like this should be able to convert a sigmoidal, ultrasensitive response like that seen in extracts into a true all-or-none response. ${ }^{(18)}$

\section{Building a biochemical switch from feedback plus ultrasensitivity}

Recent experimental studies indicate that in oocytes, the MAPK cascade generates an all-or-none response from graded stimuli. The MAPK response of individual oocytes to progesterone is as switch-like as a Hill equation response with a Hill coefficient of at least 42 (an astronomical number as Hill coefficients go), and the response of individual oocytes to microinjected Mos protein is almost as steep. ${ }^{(18)}$ It is likely that the MAPK-to-Mos positive feedback loop is critical for this all-or-none response; inhibiting the feedback loop by blocking protein synthesis changes the response from all-ornone to more gradually sigmoidal, like that seen in oocyte extracts.(18) The working hypothesis is that the oocyte's all-or-none biological response is generated by the MAPK cascade, and that the all-or-none biochemical response of the MAPK cascade arises from the combination of positive feedback and ultrasensitivity.

Analogous mechanisms operate in the production of the action potential in neurons ${ }^{(19)}$ and the lysis-lysogeny switch in $E$. coli infected with phage $\lambda .(20,21)$ The proteins involved are very different, and in the case of the action potential, the time scale of the switching is very different as well, but at some level the basic nature of the switch is the same. In all of these systems, the all-or-none character of the process depends on positive feedback-MAPK-dependent Mos accumulation in oocyte maturation, the voltage-dependent sodium conductance in the action potential, and the stimulation of lambda repressor transcription by the lambda repressor protein in the phage system. The positive feedback makes the systems unable to rest in intermediate states. In addition, each system possesses a mechanism for filtering out the first increments of stimulus so that the positive feedback loop is not set off by noise. In the case of oocyte maturation, the filtering mechanism is likely to be the inherent ultrasensitivity of the MAP kinase cascade; a steeply sigmoidal response filters out small stimuli. In the case of the action potential, the filtering mechanism is the nonlinearity of the initial response of the channel to a depolarization. And in the case of the lysislysogeny switch, the filtering is probably provided by a dimerization requirement and cooperativity. This way of constructing a switch from positive feedback plus filtering crops up again and again in cell signaling. Several examples are shown in Table 1.

\section{Building another biochemical switch}

The MAPK cascade is not the only switch in the maturation circuitry; Cdc2 is probably activated in an all-or-none fashion as well. As was the case with MAPK, the all-or-none character of Cdc2 activation is likely to depend on the fact that Cdc2 is embedded in a positive feedback loop. The seminal discovery here was the observation that the addition of cyclin B protein to Xenopus egg extracts increases the rate of $\mathrm{Cdc} 2$ activation and decreases the rate of Cdc2 inactivation. ${ }^{(22)}$

Several mechanisms may serve to filter out small stimuli from the Cdc2 activation loop, thereby establishing a threshold for activation. Cdc25 apparently dephosphorylates (and hence activates) Cdc2 by a two-step, nonprocessive mechanism, as inferred from the timing of Cdc2 tyrosine- and threonine dephosphorylation during starfish oocyte maturation.(23) Thus, holding Wee1/Myt1 constant and ignoring feedback for the moment, the steady state level of active

868 BioEssays 21.10 
TABLE 1. Mechanisms Underpinning Various All-or-None Phenomena

\begin{tabular}{|c|c|c|c|}
\hline Process & Time scale & $\begin{array}{l}\text { Mechanism for } \\
\text { positive feedback }\end{array}$ & $\begin{array}{l}\text { Mechanism for filtering } \\
\text { out small stimuli }\end{array}$ \\
\hline Action potential & Submillisecond & $\begin{array}{l}\text { Protein conformation change-depolariza- } \\
\text { tion causes increased sodium conduc- } \\
\text { tance, which causes further depolariza- } \\
\text { tion(19) }\end{array}$ & $\begin{array}{l}\text { Nonlinearity of the initial response of the } \\
\text { channel }\end{array}$ \\
\hline Mitosis (prometaphase onset) & Minutes & $\begin{array}{l}\text { Phosphorylation and dephosphorylation- } \\
\text { Cdc2 activation brings about activation } \\
\text { of its activator Cdc25 and inhibition of its } \\
\text { inhibitor Weel(22) }\end{array}$ & $\begin{array}{l}\text { Stoichiometric inhibitor? Multistep dephos- } \\
\text { phorylation mechanism for the activa- } \\
\text { tion of Cdc2? }\end{array}$ \\
\hline Xenopus oocyte maturation & Tens of minutes & $\begin{array}{l}\text { Translation control-newly synthesized } \\
\text { Mos activates the MAPK cascade, } \\
\text { which brings about stabilization of the } \\
\text { Mos }^{(14-16)} \text { (with other details of the pro- } \\
\text { cess still to be worked out) }\end{array}$ & $\begin{array}{l}\text { High Hill coefficient }(\sim 5) \text { for the response } \\
\text { of MAPK to Mos, probably as a result of } \\
\text { multistep phosphorylation of MAPK plus } \\
\text { other factors }{ }^{(8,18)}\end{array}$ \\
\hline $\begin{array}{l}\text { Lysis-lysogeny in the infection of } E \text {. coli by } \\
\text { phage lambda }\end{array}$ & Tens of minutes & $\begin{array}{l}\text { Transcriptional control- the lambda } \\
\text { repressor stimulates its transcription }{ }^{21}\end{array}$ & $\begin{array}{l}\text { Dimerization requirement; cooperativity; } \\
\text { other factors } ?^{21,25}\end{array}$ \\
\hline Myogenesis & Hours & $\begin{array}{l}\text { Transcriptional control-myoD and myo- } \\
\text { genin can up-regulate their own (and } \\
\text { each other's) expression }{ }^{(26)}\end{array}$ & $?$ \\
\hline
\end{tabular}

Cdc2 should be a sigmoidal function of the Cdc25C activitythe response should be intrinsically ultrasensitive. Moreover, a cascade of protein kinases appears to lie upstream of Cdc25C (see Fig. 1); thus, any ultrasensitivity generated by these kinases individually would be expected to combine multiplicatively, potentially yielding a highly ultrasensitive response well suited for stabilizing the Cdc25C/Cdc2 positive feedback loop. These ideas await experimental testing.

The main difference between Cdc2 and MAPK as a biochemical switch is in the length of time that the switch stays on. Cdc2 activation brings about cyclin destruction, interrupting the positive feedback loop and bringing about Cdc2 inactivation. This on-followed-by-off behavior is analogous to the action potential, where the voltage-sensitive sodium channel opens and then closes in response to depolarization. In contrast, the Mos/MEK1/MAPK loop has no autodestruct mechanism. The switch stays on indefinitely in the maturing oocyte and is only turned back off when an external influence, fertilization, brings about Mos destruction, thus interrupting the loop.

The oocyte's two switches, the Cdc2 system and the MAPK system, each at least partially control the activation of the other (Fig. 1). This arrangement may make it more difficult for either of the switches to be inadvertently tripped.

\section{Why is the response graded at the level of a population of oocytes?}

It makes biological sense that a frog should be able to induce maturation in different numbers of oocytes depending on the biological conditions, but how is this variability produced biochemically? A clue comes from the observation that oocytes are more variable in their responses to progesterone than they are in their responses to microinjected Mos. ${ }^{(18)}$ Thus, most of the variability seems to arise upstream of the MAPK cascade. Oba and co-workers have recently shown that the number of binding sites on medaka oocytes for the fish maturation-inducing hormone $17 \alpha, 20 \beta$-dihydroxy-4pregnen-3-one rises sharply during the late stages of oogenesis and falls rapidly during maturation. ${ }^{(24)}$ It seems plausible that Xenopus oocytes might vary in their progesterone receptor surface concentrations and that this variability might account for their variable sensitivity to progesterone.

\section{Conclusions}

A plausible model can now be constructed to explain why an oocyte switches between discrete cell fate states. Two key regulators of oocyte maturation, Cdc2 and p42 MAPK, are embedded in positive feedback loops that, in principle, should preclude intermediate responses. Positive feedback loops need some sort of mechanism for filtering out noise, and this is probably provided by the intrinsic ultrasensitivities of the xPlkk1/PIx1/Cdc25/Cdc2 and Mos/MEK1/p42 MAPK cascades. A variety of mechanisms, including multistep phosphorylation reactions, zero-order ultrasensitivity, and stoichiometric inhibitors probably contribute to this filtering. Building a switch from positive feedback plus ultrasensitivity seems to be a common theme in cellular regulation.

\section{Acknowledgments}

I thank Angus MacNicol for generously sharing information and preprints, Rick Aldrich, Mark Ptashne, and Dennis Thron for helpful discussions, and members of my laboratory group for suggestions on the manuscript. 


\section{References}

1. Ferrell JE Jr. Xenopus oocyte maturation: new lessons from a good egg Bioessays 1999;21:833-842.

2. Ruderman JV. MAP kinase and the activation of quiescent cells. Curr Opin Cell Biol 1993:5:207-213.

3. Gotoh Y, Nishida E. The MAP kinase cascade: its role in Xenopus oocytes, eggs and embryos. Prog Cell Cycle Res 1995;1:287-297.

4. Ferrell JE Jr. Tripping the switch fantastic: how a protein kinase cascade can convert graded inputs into switch-like outputs. Trends Biochem Sci 1996;21:460-466.

5. Brown GC, Hoek JB, Kholodenko BN. Why do protein kinase cascades have more than one level? Trends Biochem Sci 1997;22:288

6. Ferrell JE Jr. How responses get more switch-like as you move down a protein kinase cascade. Trends Biochem Sci 1997;22:288-289.

7. Kholodenko BN, Hoek JB, Westerhoff HV, Brown GC. Quantification of information transfer via cellular signal transduction pathways. FEBS Lett 1997;414:430-434.

8. Huang C-YF, Ferrell JE Jr. Ultrasensitivity in the mitogen-activated protein kinase cascade. Proc Natl Acad Sci USA 1996;93:10078-10083.

9. Chen M, Cooper JA. The beta subunit of CKII negatively regulates Xenopus oocyte maturation. Proc Natl Acad Sci USA 1997;94:9136-9140.

10. Chen M, Li D, Krebs EG, Cooper JA. The casein kinase II beta subunit binds to Mos and inhibits Mos activity. Mol Cell Biol 1997;17:1904-1912.

11. Burack WR, Sturgill TW. The activating dual phosphorylation of MAPK by MEK is nonprocessive. Biochemistry 1997;36:5929-5933.

12. Ferrell JE Jr, Bhatt RR. Mechanistic studies of the dual phosphorylation of mitogen-activated protein kinase. J Biol Chem 1997;272:19008-19016.

13. Howard EL, Charlesworth A, Welk J, MacNicol AM. The MAP kinase signaling pathway stimulates Mos mRNA cytoplasmic polyadenylation during Xenopus oocyte maturation. Mol Cell Biol 1999;19:1990-1999.

14. Gotoh Y, Masuyama N, Dell K, Shirakabe K, Nishida E. Initiation of Xenopus oocyte maturation by activation of the mitogen-activated protein kinase cascade. J Biol Chem 1995:270:25898-25904.

15. Matten WT, Copeland TD, Ahn NG, Vande Woude GF. Positive feedback between MAP kinase and Mos during Xenopus oocyte maturation. Dev Biol 1996;179:485-492

16. Roy LM, Haccard O, Izumi T, Lattes BG, Lewellyn AL, Maller JL. Mos proto-oncogene function during oocyte maturation in Xenopus. Oncogene 1996;12:2203-2211.

17. Huang C-YF, Ferrell JE Jr. Dependence of Mos-induced Cdc2 activation on MAP kinase function in a cell-free system. EMBO J 1996;15:21692173

18. Ferrell JE Jr, Machleder EM. The biochemical basis of an all-or-none cell fate switch in Xenopus oocytes. Science 1998;280:895-898.

19. Kandel E, Schwartz J, Jessell T. (ed): Principles of neural science. 3rd Ed. Norwalk, CT: Appleton \& Lange; 1991. p 104-118.

20. Ptashne M, Johnson AD, Pabo CO. A genetic switch in a bacterial virus. Sci Am 1982;247:128-130, 132, 134-140.

21. Ptashne M. A genetic switch: phage $\lambda$ and higher organisms. Cambridge MA: Cell Press and Blackwell Scientific Publications. 1992

22. Solomon MJ, Glotzer M, Lee TH, Philippe M, Kirschner MW. Cyclin activation of p34cdc2. Cell 1990;63:1013-1024.
23. Borgne A, Meijer L. Sequential dephosphorylation of p34(cdc2) on Thr-14 and Tyr-15 at the prophase/metaphase transition. J Biol Chem 1996;271: 27847-27854.

24. Oba Y, Yoshikuni M, Tanaka M, Mita M, Nagahama Y. Inhibitory guaninenucleotide-binding-regulatory protein alpha subunits in medaka (Oryzias latipes) oocytes_cDNA cloning and decreased expression of proteins during oocyte maturation. Eur J Biochem 1997;249:846-853.

25. McAdams HH, Shapiro L. Circuit simulation of genetic networks. Science 1995:269:650-656.

26. Thayer MJ, Tapscott SJ, Davis RL, Wright WE, Lassar AB, Weintraub H. Positive autoregulation of the myogenic determination gene MyoD1. Cell 1989:58:241-248.

\section{Appendix}

There are two simple ways to quantify how steeply sigmoidal a response is. The first is to assume that the responses of the cascade components are well-approximated by the Hill equation

$$
R=\frac{S^{n}}{K^{n}+S^{n}}
$$

where $R$ is the response, $S$ is the stimulus, $K$ is the $\mathrm{EC}_{50}$, and $n$ is the Hill coefficient. Then, the Hill coefficient is a measure of how steeply sigmoidal the response is. A Hill coefficient of 1 corresponds to a Michaelian response, and a Hill coefficient of greater than 1 corresponds to an ultrasensitive or switchlike, sigmoidal response. If the response of MEK1 to Mos exhibits a Hill coefficient of $m$ and the response of p42 MAPK to MEK1 exhibits a Hill coefficient of $n$, than the apparent Hill coefficient for the response of p42 MAPK to Mos will be no greater than $m \cdot n \cdot{ }^{(6)}$

Alternatively, one can define the sensitivity or response coefficient for the response $R$ to a stimulus $S$ to be

$$
\frac{\mathrm{d} \ln R}{\mathrm{~d} \ln S} \text {. }
$$

This type of sensitivity is essentially the "order" of a response, and it varies with the stimulus. From the chain rule, it then follows that the overall sensitivity of a cascade is the product of the sensitivities of the individual levels. ${ }^{(7)}$ Note that for a response described by the Hill equation, the response coefficient is equal to the Hill coefficient for small stimuli, and then decreases to zero as the stimulus increases. 